\title{
Lightweight rigidly foldable canopy using composite materials
}

\author{
Kensuke Ando ${ }^{1} \cdot$ Bunji Izumi ${ }^{2} \cdot$ Mizuki Shigematsu $^{1} \cdot$ Hiroki Tamai $^{1} \cdot$ Jun Matsuo ${ }^{3} \cdot$ Yuki Mizuta $^{3} \cdot$ Takeshi Miyata $^{4}$. \\ Jiro Sadanobu ${ }^{5} \cdot$ Kai Suto $^{6} \cdot$ Tomohiro Tachi $^{6}$ (D)
}

Received: 30 November 2019 / Accepted: 6 November 2020 / Published online: 12 November 2020

(c) The Author(s) 2020 OPEN

\begin{abstract}
This paper presents a novel origami-based portable deployable canopy system developed using fiber reinforced plastics. A modular system composed of multiple developable strips is proposed to provide a one degree-of-freedom deployment motion from a flat-folded state to a fully deployed state. Each strip is comprised of panels with embedded compliant hinges whose pattern is created in a planar configuration through the laying out of prepreg composite sheets and multi-step curing. The design process of a canopy using this system is demonstrated herein. To capture the complex behaviors and functionality, the design process involves developing different analytical models for each step starting with a simplified model and ending with a refined model. In this case, we defined a parametric design family from rigid origami theory and determined preliminary design parameters through a multi-objective optimization (MOO) scheme in order to balance performance against manufacturing constraints. We then applied geometric nonlinear analyses to assess the kinematic behaviors of the folding actions and also the buckling behavior of the structure in its deployed state. The analyses indicated the need for stability improvement, provided using tension elements. The structure was divided into developable parts that can be manufactured in a planar state. With a total mass of $27 \mathrm{~kg}$, the system can be carried by two or three persons and deployed within a minute.
\end{abstract}

Keywords Rigid origami · Deployable structure $\cdot$ Composite materials $\cdot$ Compliant hinge $\cdot$ Lightweight structure

\section{Introduction}

Portable deployable structures are ideal solutions for temporary buildings such as shelters for disaster relief or pavilions for various events. They are: (1) rapidly erectable and retractable to satisfy urgent demands; and, (2) reusable multiple times for a variety of purposes including rare events. As such, they consume fewer resources and produce fewer waste. We observed that the following properties would further maximize the effectiveness of the structures in these aspects:

1. The structure has easily controllable deployment and folding motions. The structural module is lightweight, requiring no additional equipment for erection or elements for finishing.

2. The structure is modular such that separate pieces can fit together to form various shapes and can be used in several states during deployment.

Major approaches for creating deployable structures include pneumatic structures, deployable frames (e.g. scissors mechanisms), and foldable folded plates (i.e. origami structures). Regarding property 1 , the former two mentioned solutions have an advantage in terms of their lightness. However, both require surface finishing usually composed of thin membranes. The folding behavior of the attached finishing is less controllable and leads to

Tomohiro Tachi, tachi@idea.c.u-toyo.ac.jp | ${ }^{1}$ Nikken Sekkei Ltd, Tokyo, Japan. ${ }^{2}$ Norwegian University of Science and Technology, Trondheim, Norway. ${ }^{3}$ Teijin Ltd, Tokyo, Japan. ${ }^{4} \mathrm{GH}$ Craft Ltd, Shizuoka, Japan. ${ }^{5}$ Sadanobu Science \& Technology Office, Tokyo, Japan. ${ }^{6}$ The University of Tokyo, Tokyo, Japan. 
lower efficiency in the folding stage. In contrast, origami systems offer reversible folding and deployment functions with watertight rigid surface panels, provided that the kinematics of the rigid origami is carefully examined [1-4]. This makes the system potentially more reliable in the process of folding it down into its compact stowed state. The potential downside is that the structure can be heavy if conventional plate materials and mechanical hinges are used.

In this paper, we present an origami-based deployable structure using fiber reinforced plastic (FRP) plates with compliant hinges to achieve lightness. In general, a critical issue of using composite material is their manufacturing cost-particularly the cost of creating a mold and for the manual labor; the textile has to be manually laid out on to the mold in order to create a three-dimensional surface. The approach outlined in this paper solves this issue by fabricating each developable module with compliant hinges in a flat state. The hinges are created as the FRP using a soft matrix as proposed in $[5,6,7]$. Several studies investigate the use of FRP hinge for deployable structures aimed for relatively small systems or structures under negligible gravitational load [6, 8]. Our study aims to apply such FRP-hinged systems for applications in architectural scale under gravity.
As a case study, we designed and fabricated a FRP portable origami canopy with height of $2.85 \mathrm{~m}$ that can act as an acoustic reflector and diffuser. The average thickness of the material is about $1 \mathrm{~mm}$, and the total mass of the structure is $27 \mathrm{~kg}$. The geometry of the module was chosen so that the structure is always flat-foldable and rigidfoldable after assembly with exactly one kinematic degree of freedom (DOF). This allows the structure to continuously fold down to a stowed state with simple actuation control. Together with its lightness, the canopy structure can be carried and deployed by two persons within a minute (Fig. 1).

The design and fabrication of functional transformable structures requires simultaneous considerations of geometry, functions of the space, kinematics, stability, materials, and fabrication. To tackle this multi-modal design process, we formed an interdisciplinary team of comprised of structural engineers and architectural designers (the first four authors, Nikken), specialists in composite materials (fifth, sixth, and eighth authors, Teijin, Sadanobu Science \& Technology Office), engineers of composite material fabrication (seventh author, GH Craft,) and scientists in origami geometry (last two authors).

There is no established design process or unified analysis models for creating folding-based ultra-thin structures

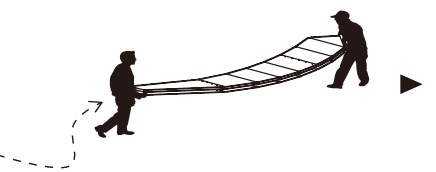

01 carry

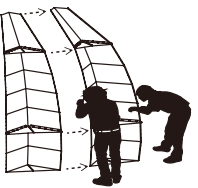

02 build

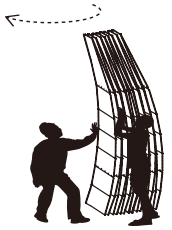

03 deploy

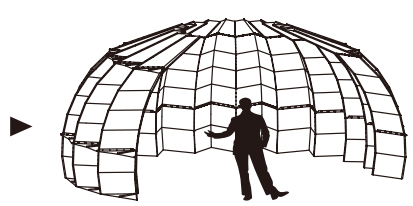

04 complete

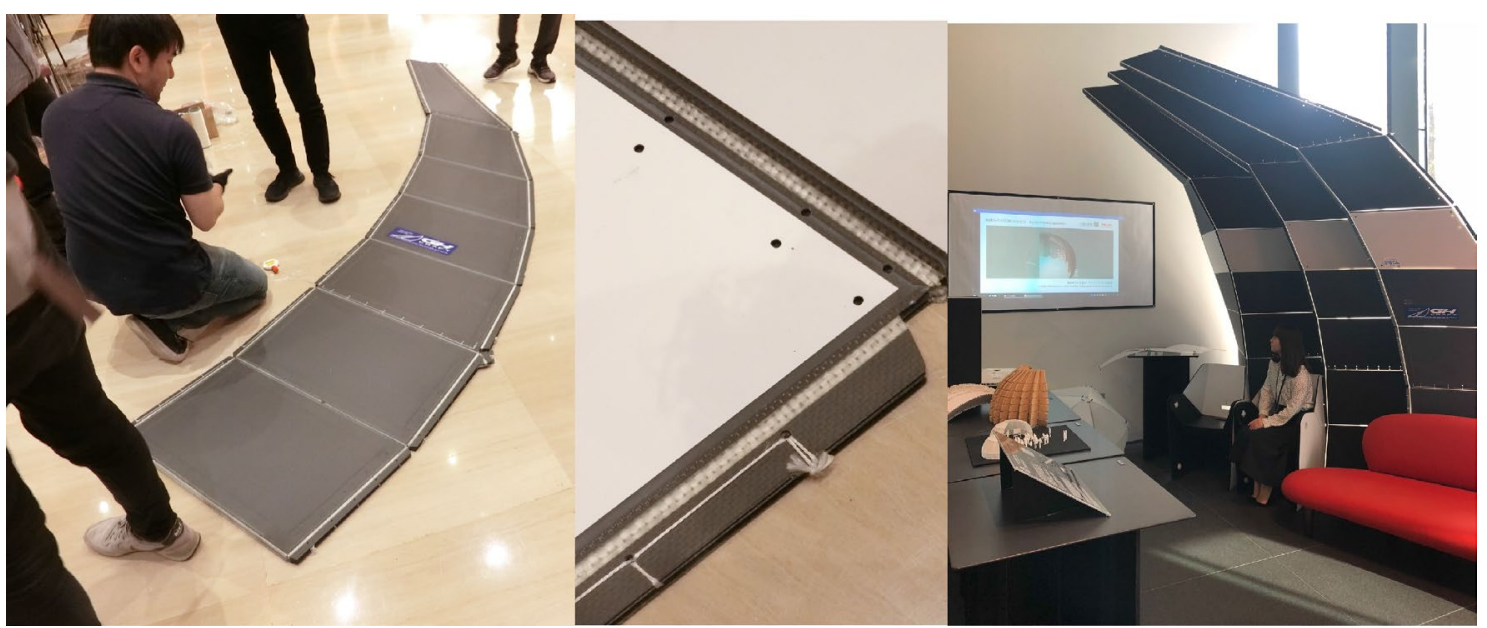

Fig. 1 Deployable origami canopy. Top: the concept illustration. Bottom: actual structure (left: folded planar state; middle: the details of joints and hinges; right: the deployed state in use.)

\section{SN Applied Sciences}


with compliant hinges. We propose a design process utilizing various simulation models for different steps. Beside the mathematical rigid origami kinematics model, different types of models are developed for the physical simulations, including structural behavior, starting from a simplified model and ending with a much more refined model. First, we begin from geometric concepts based on rigid origami kinematics to identify the parametric family of rigid-foldable and flat-foldable modular structures (Sect. 2). Then, in the second step, we determine the preliminary shape with proportions that balance the tradeoff between structural stiffness, sound diffusion and solar energy gain through a multi-objective optimization process (Sect. 3). In order to assess the folding and deployment behaviors, a wireframe model under gravity is used (Sect. 4) to identify the bifurcation folding behavior, leading to improvements in the design. In Sect. 5 of this paper, we perform geometric nonlinear load incremental analyses as well as eigenvalue buckling analysis with thin shell finite elements and elastic hinges connecting the panels to identify buckling behaviors under the self-weight of the system. Based on the findings in the stability study, tension elements (i.e. stiffeners) are added to the design to prevent buckling. Section 6 covers the manufacturing process which incorporates composite origami materials into the design of the modules. We show the details of builtin compliant hinges with soft matrix and assembly of the modules by special tie methods. Finally, Sect. 7 concludes the paper and shows our future vision of origami-based composite deployable systems.

\section{Parametric family from rigid origami geometry}

We introduce a modular system that creates a family of flat-foldable and rigid-foldable surfaces with exactly 1-DOF rigid-folding motion. Such a one-DOF origami can be constructed by using pattern with quadrangular panels [2-4]; however, in general, origami structures composed of quadrilateral panels are over-constrained, so they need to have intrinsic symmetry to form a mechanism. To create over-constrained mechanisms, we use tubular rigidly foldable origami symmetrically generated from a polygonal cross section with parallel extrusion [3, 9].

The geometry of the system is based on part of a large torus which, by its assembly, can be used to create various forms/arrangements e.g. a covered corridor (Fig. 2). Five steps are needed to construct a rigid-foldable and flatfoldable origami torus. In particular, the second and third steps guarantee that these characteristics can be achieved (Fig. 3).

1. We construct the torus defined by radiuses of smaller and bigger circles, defined as $r$ and $R$, respectively. We set $X Y Z$ axes such that the bigger circle lies on $X Y$ plane, the smaller circle lies on $X Z$ plane, and the origin lies on the center of torus.

2. Construct a section polyline $P$ on the small circle. A parallelogram strip is formed by extruding $P$ along

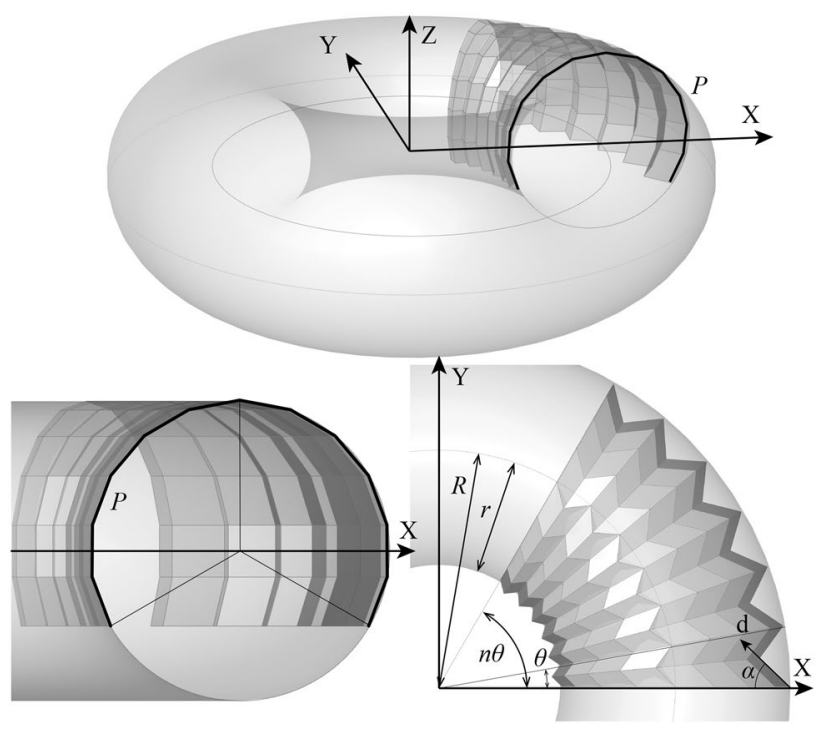

Fig. 3 Geometric parameters defining the shape

Fig. 2 Flat-foldable and rigidfoldable covered corridor. Each module approximates part of a torus
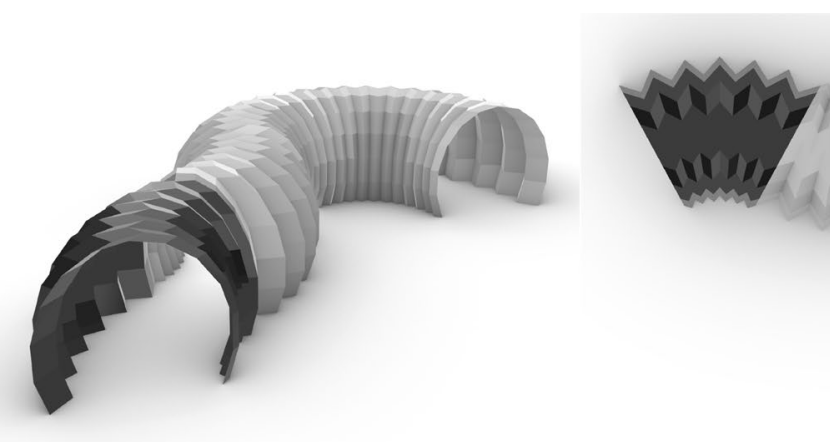
direction $\mathbf{d}$ on $X Y$ plane. The angle between $\mathbf{d}$ and $-X$ axis is denoted by $\alpha$. The choice of $\alpha$ is important for the stiffness of the structure. For more detailed discussion, see Sect. 4.

3. The strip obtained by step 2 is trimmed by a trimming plane, defined as the rotation of $X Z$ plane around $Z$ axis by an angle of $\frac{\theta}{2}$.

4. Mirror copy the trimmed parallel trapezoidal strip with respect to the trimming plane.

5. Concatenate $n$ rotated copies of the strip constructed in steps 3 and 4 around the $Z$ axis.

(Design parameters are $r, R, \theta, a, n, P$ ).

The torus surface constructed as above has a 1-DOF rigid folding motion. This folds flat because of the mirror symmetry used in step 4 . Another valuable property is that all the horizontal lines of the parallelograms are always parallel to the $X Y$ plane during the folding motion.

If $P$ is mirror-symmetric with respect to an axis parallel to the $Z$ axis, (i.e., arch shape), the legs always sit on the ground. This leads to stability while folding. Also, the structure serves as a pavilion at any interval of the deployment motion. Since each extruded strip is developable, this forms the basis for module manufacturing. This modular design has advantages as the assembly of a single type of module can be used to create various configurations including a fully closed structure, corridor, and open canopy.

Figure 4 shows the rigid folding motion. The top part of the structure gets expanded until it becomes flat, at which point, the mountain and valley of the creases get reversed. At this critical point, the angle $\theta$ projected to the $X Y$ plane hits its maximum. This results in the bifurcation behavior

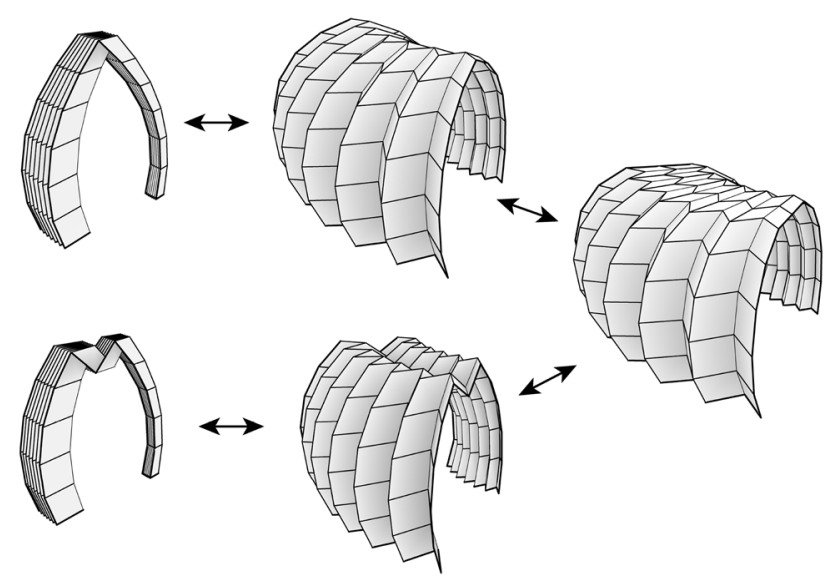

Fig. 4 Folding motion of a module. The top part gets reversed at the critical folded state at the far right as described in Sect. 4, which also describes measures to avoid such behavior.

\section{Preliminary form finding though Multi-Objective Optimization (MOO)}

The canopy has multiple potential uses: as (1) a self-standing structure; (2) a sound reflector to surround the speaker on the stage; and, (3) a solar energy collector. The solar energy collected by Photovoltaic (PV) panels attached to the canopy can be used as a supplementary energy source for outdoor use such as an outdoor stage or a temporary shelter for refugees in the event of a natural disaster.

We obtain preliminary design parameters with balanced overall performance measured in terms of (1) structural stiffness, (2) homogeneity of the reflection of speaker's voice, and (3) reception of the solar radiation by the exterior side of the panels. Multi-objective optimization (MOO) is used to evaluate the best balance of these different performance measurements. Rhino/Grasshopper was used to perform parametric design, analyses, and optimization. Octopus 0.3.6 [10] was used to find the Pareto optimal solutions. HypE algorithm [11] was chosen among the options within Octopus multi-objective optimization platform.

\subsection{Design parameters and constraints}

The design parameters $r, R, \theta, \alpha, n$, as defined in Sect. 2 are used. For the polyline section $P$, we focused on a cantilever shape, considering the usage as a sound reflector, so $P$ was set to equally divide an arc of the small circle defined by two angles (angles 1 and 2 in Fig. 5). The design parameters are constrained by the maximum size of each developable strip allowed for in fabrication, as well as the required minimum size of the enclosing space.

\subsection{Objective functions}

\subsubsection{Stiffness of structure}

The design intent is to minimize the displacements under vertical loads. Linear static structural analyses were undertaken assuming that all folded panels are connected with rigid joints. These linear analyses neither consider the folding transformation along the creases, nor buckling effects (which are instead examined in the later nonlinear analyses in Sects. 4 and 5). Here, our focus was on preliminary selection of a balanced shape for initial design. The structural engineering tool Karamba 1.3.0 [12] was used. Structural 


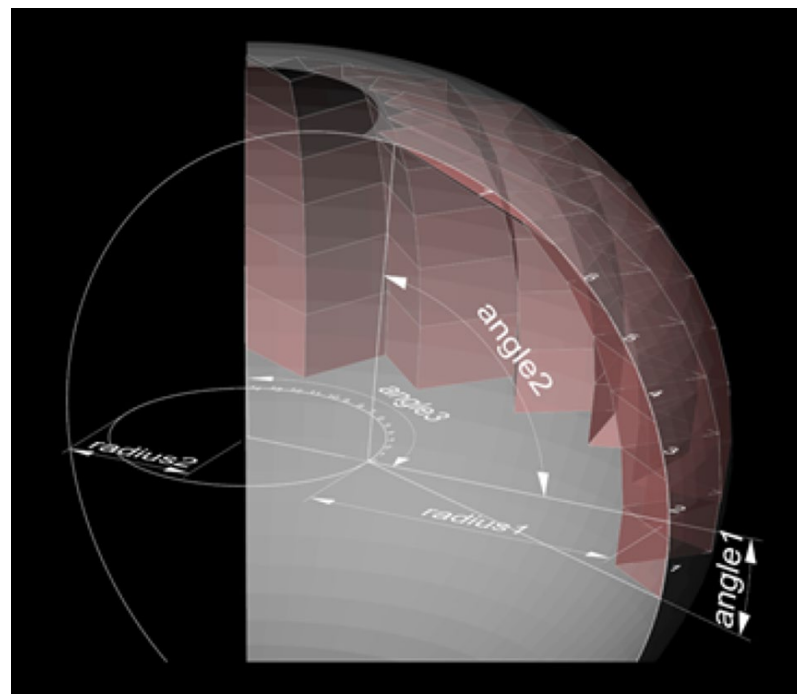

Fig. 5 Design parameters of canopy for MOO

elements employed in this Section were modelled using a 2-dimensional mesh in Grasshopper, which were then interpreted as shell elements in Karamba. The shell elements consist of 3 nodes with 6 DOFs and its shell element formulation is based on the TRIC element [13], except that the shell element in Karamba is based on the Kirchhoff theory.

The material properties used are as follows: modulus of elasticity is $65,000 \mathrm{~N} / \mathrm{mm}^{2}$, Poisson's ratio is 0.3 ; and, the density is taken as $1550 \mathrm{~kg} / \mathrm{m}^{3}$. The thickness is assumed to be $1 \mathrm{~mm}$.

\subsubsection{Homogeneity of voice distribution}

The homogeneity evaluates how the sound will be reflected on the panel and conveyed to the audience if a person speaks in front of the structure. Since the structure is composed of many panels with different angles, its acoustic performance was evaluated using a ray tracing method. Figure 6 shows the overall set-up of the simulation. A point sound source was placed in front of the structure at about the height of a human head. Rays representing sound travel were released non-directionally. The first and second reflections are used to calculate where the sound will arrive in the area of the audience, represented by the inclined rectangular surface. The surface was divided into sub-areas, and the number of hits were counted to measure the density of the power level. The variance of such distribution was minimized. The code was written in the Grasshopper software environment by the second author [14].

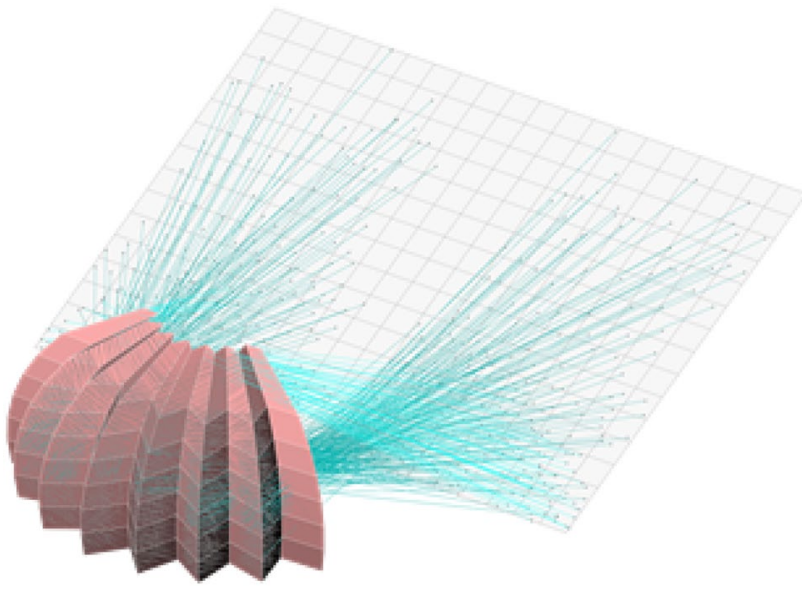

Fig. 6 Acoustic simulation based on ray tracing

\subsubsection{Maximizing reception of solar radiation}

The last objective is to maximize the reception of solar radiation onto the structure. At the same time, with the same objective function the shape can maximize the solar shielding effect combined with heat shielding paint. Unfortunately, these functions associated with solar radiation were not implemented to the real-scale model. However, the study does demonstrate the potential benefit of applying multi-objective design approach by MOO (Fig. 7). Ladybug [15] was used to carry out solar radiation simulation.

\subsection{Finding the balanced design}

The objective functions mentioned in the previous sections tend to trade off with each other. For instance, a shape with deep folds, i.e. smaller angles $\alpha$, has better structural performance since stiffness increases with the folded plate effect, whilst it has less reception of

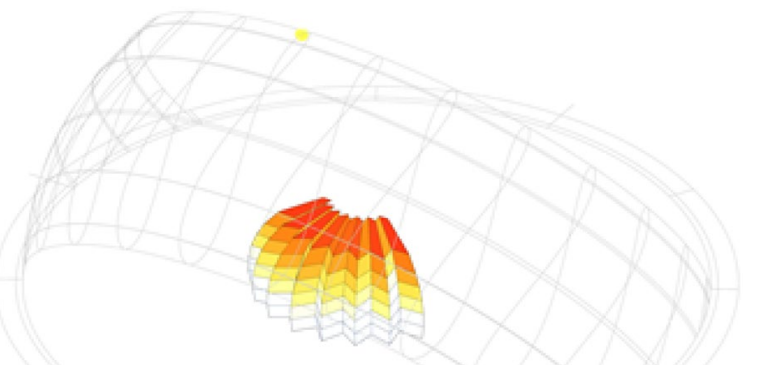

Fig. 7 Solar radiation simulation. Saturated color indicates the areas exposed to higher solar power 


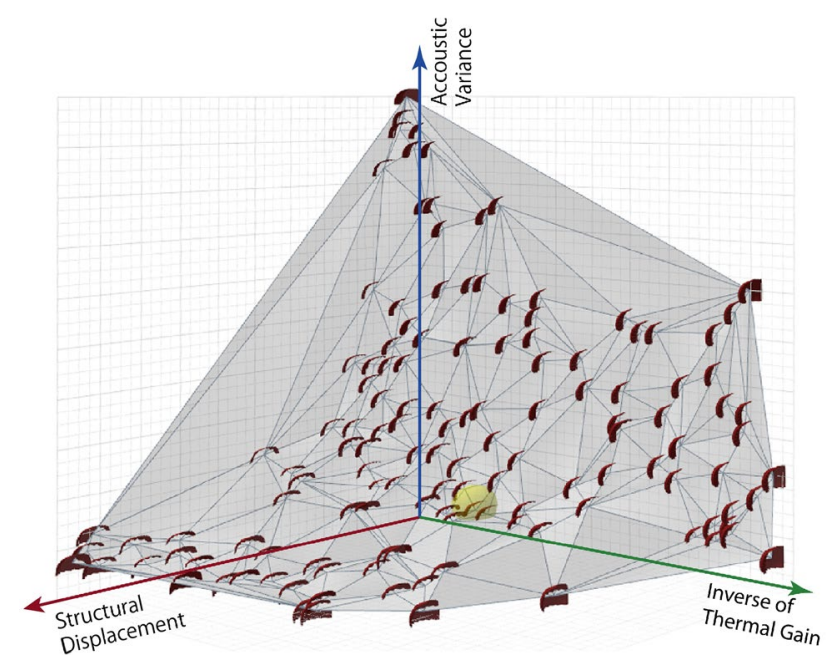

Fig. 8 Instances on Pareto-front surface

solar radiation because the incident angles of solar rays become too shallow for some panels. Our design goal was to seek the best-compromise of each of the three contradicting objectives within acceptable ranges.

Figure 8 shows solution configurations on an obtained Pareto-front of the three objectives, "structural displacement", (inverse of) "thermal gain" and "acoustic variance". Each instance on the Pareto front has the property that none of the objectives can be improved without compromising any others. The instance highlighted in yellow in Fig. 8 was selected for further nonlinear structural analyses and fabrication-aware design.

\section{Deployment Simulation through Wireframe Model}

The transformation of the origami canopy in rigid origami model was simulated to see how it would dynamically fold and unfold whilst under gravity loading. Mechanical mobilization using actuators was originally entertained during design as well as using manual operation by a few people. Thus, the investigation was made with various assumptions on how the supports are constrained or guided during operation, which points are actuated, and the speed of the actuators. At the end, the authors did not proceed with a design using mechanical mobilization, but this investigation provided a few important findings that lead to the design improvements, including most notably undesirable bifurcation folding behavior.

\subsection{Octahedral wireframe model}

The kinematics of rigid origami can be simulated either by rigid bodies connected by hinges (angular model) [16] or bars connected along edges (truss model) [17-19]. In the truss model, with assumed infinitely stiff bars, the rigidity of a non-triangular panel is either modeled by triangulation with rigid joints $[18,19]$ or by polyhedralization. We employed the polyehdralization scheme: each rigid panel was modeled with thirteen bars constituting a squashed octahedron with a short central strut. In order to understand the kinematic behavior using time integration dynamic analysis with actuator elements as well as by DOF analysis applying singular value decomposition, the latter polyhedral (octahedral) truss models is most preferable.

The articulation between the panels was assumed to be provided by linear hinges and modeled simply by sharing an edge of adjacent squashed octahedra (Fig. 9), i.e. no rotational stiffness. The actuator is modeled with a bar element that can be shortened or elongated in each time step. Direct integrated time history analysis is applied with a geometric nonlinear formulation for bar elements [22]. With this idealized modeling, it was possible to quickly investigate the transformation process by actuators without resorting to time-consuming calculations with very thin shell elements. It also allowed easy switching between dynamic analysis and DOF analysis.

This study, in fact, made us notice that overhang facets could cause bifurcation folding. That is to say that they can go either downward or upward depending on the balance of the speed of operation as well as the weight of the facets (Fig. 10a). Obviously, this would make the control of the transformation more difficult. The cause of this issue comes from the fact that the DOF is localized at one position and the actuator loses its effect. Although this behavior seems to contradict single-DOF behavior, one should note that there may be more than two ways of folding with a single-DOF system. Based on this finding, the design was modified to remove the crown point of the reference torus so that such bifurcating conditions cannot occur (Fig. 10b). Section 4.2 further discusses on this with the results of DOF analysis.
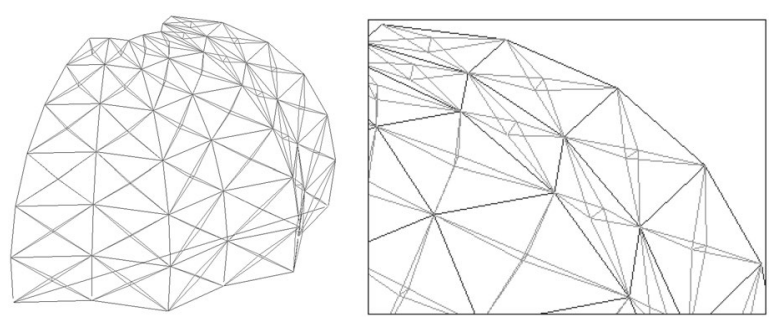

Fig. 9 Wireframe dynamic model and enlarged image in frame 

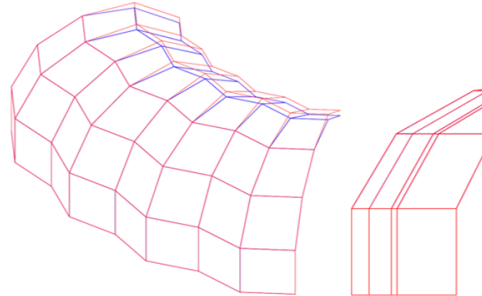

The second fold pointing upward

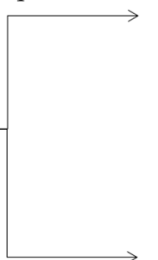

The second fold pointing downward
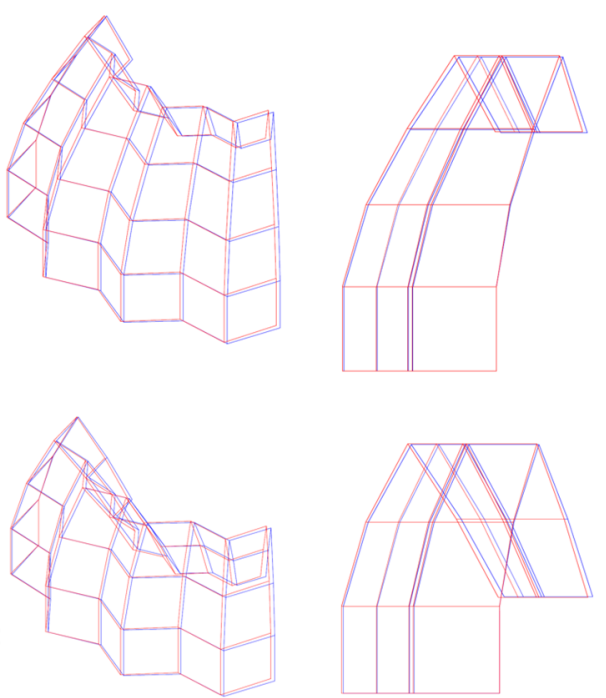

(a) Bifurcated fold in transformation represented by null space vector displacement (red) over evaluated positions (blue)
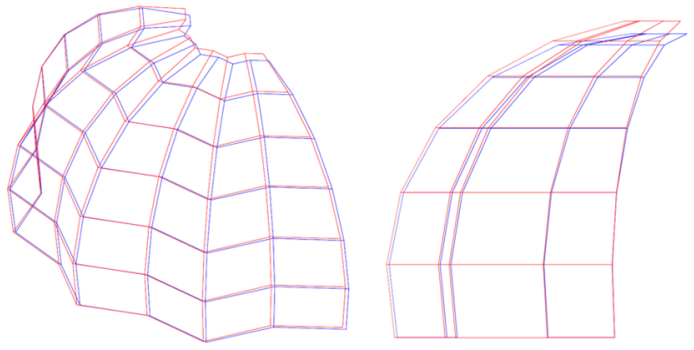

Unique fold
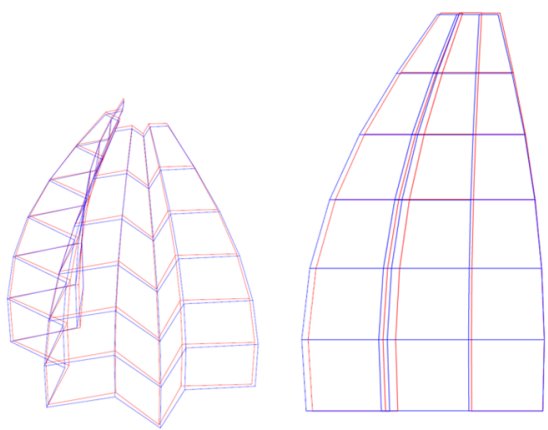

(b) Unique fold in transformation represented by null space vector displacement (red) over evaluated

positions (blue)

Fig. 10 a Bifurcated fold in transformation represented by null space vector displacement (red) over evaluated positions (blue) b Unique fold in transformation represented by null space vector displacement (red) over evaluated positions (blue)

\subsection{Calculation of DOF and notes on fold bifurcation}

In order to investigate the fold bifurcation problem, the DOF of the canopy structure, represented by a model with wireframe octahedra, was calculated by finding the nullspace of the Jacobian matrix of the bar lengths as functions of the global coordinates of the nodes. DOFs were calculated at several transitional positions in a series of transformation. As shown in Fig. 10a, b in all the positions calculated, including the above-mentioned bifurcation point, the applied geometry allows only one DOF to the structure. In the figures, the shapes in blue are the actual positions evaluated, and the red ones represent transformable direction from positions calculated as null-space vectors.

It is noteworthy that at the point of fold bifurcationthe moment when the panels in the second row from top become precisely flat-the DOF remains one, but the freedom is localized to the folding line. The position shown on the left side in Fig. 10a) is in such a condition. In contrast to those on the right side of Fig. 10a), where all apexes of the structures move along unique trajectories at the same time, the one in the bifurcation position folds only along the second hinge line from the top. This means that 
a horizontal actuator cannot control the transformation at this point because there is no movement in the horizontal direction, and that the pointing direction at this moment dictates the shape of fold that proceeds. Therefore, we would need more than one linear actuator for such a case, even if it is a strictly single-DOF system. This bifurcation behavior contrasts with the kinematic bifurcation caused by the branching of the configuration space [9, 23, 24]; in the branching case, the Jacobian matrix degenerates, and the computed infinitesimal DOF exceeds one. The bifurcation observed in the structure can be interpreted as the cusp point of the configuration space when they are projected to the lower dimensional space parameterized by the actuator configurations, so they are dependent on where the actuators are assigned.

The diagram in Fig. 11 illustrates such fold bifurcation. For any position during the transformation prescribed, a unique distance travelled by the horizontal actuator is determined. However, for a stroke distance prescribed, there are two possible positions depending on which path it comes. In other word, at the maximum bound of the travel distance, i.e. fully open state, bifurcation occurs.

\section{Stability analysis with shell finite elements}

Although a rigid folding transformation was assumed in the conceptual design phase, the panels are, in reality, very thin and elastic. Thus, the stability of the canopy with the panel bending behavior needs to be examined. Static behaviors of the canopy in the expanded configuration were investigated under its own weight and material properties by utilizing eigenvalue buckling analysis and geometric nonlinear analysis. The structural investigations focused on the stability of the articulated thin-panel structure with limitations in determining the loose property of

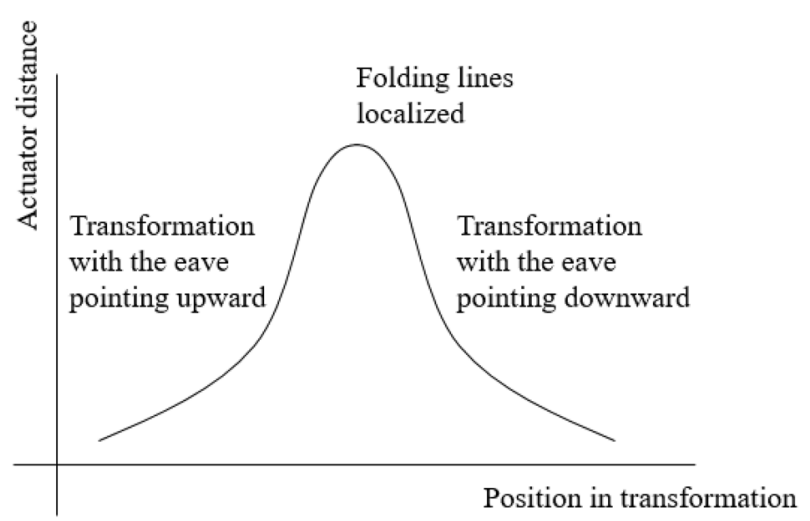

Fig. 11 Conceptual sketch of transformation path the "watoji" joints (described in Sect. 6). This investigation lead to the design improvement such that the stability is increased by deepening the crease of folds, effective support conditions and by adding foldable web-elements in the valleys of folds.

\subsection{Description of structure and analytical model}

The stability relies totally on the shape of the structure because the panel's thickness ratio (ratio of length to thickness) is extremely large. The ratio is in the order of 1000 for some panels, and it could be even larger. The shape of the structure can be seen as a part of a dome, but it is clear that the shell effects of a dome shape cannot be expected unless it is fully closed. Rather, the folded plate effects contribute to the stiffness of the stand-alone cantilever, while the angles at the articulations contribute to the local stability of the thin panels by shell-like effects.

An origami-based system is expected to exhibit a complex elastic behavior. Specifically, by the coupling of folding and elastic bending, the localized folding and unfolding becomes prominent even if the structure is kinematically a one-DOF mechanism in the rigid origami model $[25,26]$. Furthermore, the localized unfolding of creases reduces the expected stiffness of the folded plate system as load increases; the reduced stiffness results in the snap-through buckling $[27,28]$. A bar-and-hinge model is reported to be effective $[18,28]$ to qualitatively capture the nonlinear behavior of origami; however, the previous works assume the thickness ratio of the order of 100 , which is 10 times thicker than our model. Additionally, the compliant hinges and lacing-based joints in the structure are expected to have a part to play in the stability. To model the system with ultra-thin panels connected by the compliant hinges, we built up our computational model using thin shells with springs as described in the following sections.

\subsection{Static analysis for stability investigation}

Geometric nonlinear calculations with thin shell elements was performed using the commercial structural analysis program SOFiSTiK [29], in order to evaluate the structural behavior of the canopy. The panels made of shell finite elements were linked with two sets of spring elements that simulate the embedded hinges integrated with resin and mechanical hinges coupled by "watoji" lacing. The resinintegrated ones are used for horizontal articulation of the modules with a range of rotation up to $15^{\circ}$ and made stiffer than the other for stability. On the other hand, the hinges combined with "watoji" were made softer for easier transformation that requires a range of rotation up to $50^{\circ}$. 
The latter have looseness due to fabrication tolerances and nature of hand-lacing.

The stiffness of the joints were modeled using three linear (axial) springs for the axial, in-plane and out-of-plane shear directions, and one rotational spring for out-of-plane bending. Table 1 shows the coefficients of the springs with a length of $1 \mathrm{~mm}$ assumed in the model per one-meter breadth.

The stiffness values shown above were estimated by structural analysis prior to prefabrication. Those in the integrated hinges were set equal to a strip of $0.1 \mathrm{~mm}$ thick plate, while actual panel thickness ranges from $0.5 \mathrm{~mm}$ in the upper parts, $0.9 \mathrm{~mm}$ in the middle parts, to $1.3 \mathrm{~mm}$ at the lower parts as shown in Fig. 17. The hinges coupled by "watoji" lacing undergo larger deformations. Small samples were made to see how flexible they were. The order of the rotational spring coefficient was set to as low as one tenth of the integrated one, which is almost a simple hinge with no stiffness. These estimations push the limits of elastica theory for such large deformations but were accepted for this project considering the scale and experimental usage of the canopy. The modeling of looseness in the "watoji" lacing was translated to softening of the axial and shear stiffness arbitrarily to the order of $1 \%$ of the integrated joint for the sake of convenience. It must be noted that the goal of this investigation focused more on collecting information that leads to design improvement by understanding the structural behavior within certain boundaries of assumptions than thorough computational evaluation of this kind of structure.

The stability of the structure was investigated by utilizing eigenvalue buckling analysis as well as load incremental analysis with geometric nonlinearity. The following subsections discuss the analysis results from the models before and after the design modifications. No initial imperfection was applied to geometry in the analyses. The authors abandoned applying initial imperfections from the investigation without having established the accurate joint modeling.

\subsubsection{Analysis results of the initial geometry}

The first model was selected from the Pareto set filtered by multi-objective optimization and was examined closely by the structural analysis techniques described above. It was made with only the integrated hinges as the use of "watoji" lacing had not been conceptualized yet. The model was supported vertically at all folding points and only two outermost points were horizontally supported. See Fig. 12a).

The analysis results showed that the structure was very flexible due to the high thickness ratio as well as hinges with very low stiffness. Both eigenvalue buckling analysis and load incremental analysis on the original model indicated that the lower end panels would undergo local buckling. Figure 12b) shows the lowest buckling mode with a factor of 0.13 to its self-weight, and Fig. 12c) shows deformation under the limit load by a factor of 0.07 in the incremental analysis. The stability of the original configuration turned out to be critically below self-supporting requirements.

Both phenomena are thought to be associated with instability of the bottom end panels. The deformation under the incremental load analysis indicated very well that the structure tends to lose the stiffening effects of the shape as it deforms under its own weight. The panels at the bottom edges were critically stressed in compression under the cantilever action of the canopy. The out-of-plane deformations of these panels, which can be seen as local buckling, tend to squash the ridges and valleys without horizontal restriction of the supports. They effectively flatten the folded plate system and increase the deformation further. Such a folding-and-bending-coupled behavior is unique to in the origami-based structure with thin panels, and is worth future investigation.

Recall that, in theory, a rigid origami system with single DOF can deform only in the direction of the DOF. It means that the system does not yield any large deformation (i.e. stable) once the last DOF is constrained. However, the analysis with an elastic model demonstrated otherwise. This concluded that the stability of the origami structure cannot be relied on constraining the last DOF if elastic deformation of the origami panel is significant.

\subsubsection{Analysis results of the modified geometry}

In the previous model, the angle was too shallow to contribute to the structural stability. In order to improve the stability, MOO was applied again with increased variable
Table. 1 Spring stiffness values used for linear joints between panels

\begin{tabular}{lllll}
\hline Spring direction & Axial & In-plane shear & Out-of-plane shear & $\begin{array}{l}\text { Out-of- } \\
\text { plane } \\
\text { bending }\end{array}$ \\
\hline Unit per breadth & $\mathrm{kN} / \mathrm{m}$ & $\mathrm{kN} / \mathrm{m}$ & $\mathrm{kN} / \mathrm{m}$ & $\mathrm{kNm} / \mathrm{rad}$ \\
Integrated hinge & $6.5 \times 10^{6}$ & $2.5 \times 10^{6}$ & $2.5 \times 10^{6}$ & $5.4 \times 10^{-3}$ \\
Watoji joint & $6.5 \times 10^{4}$ & $2.5 \times 10^{4}$ & $2.5 \times 10^{4}$ & $5.4 \times 10^{-4}$ \\
\hline
\end{tabular}




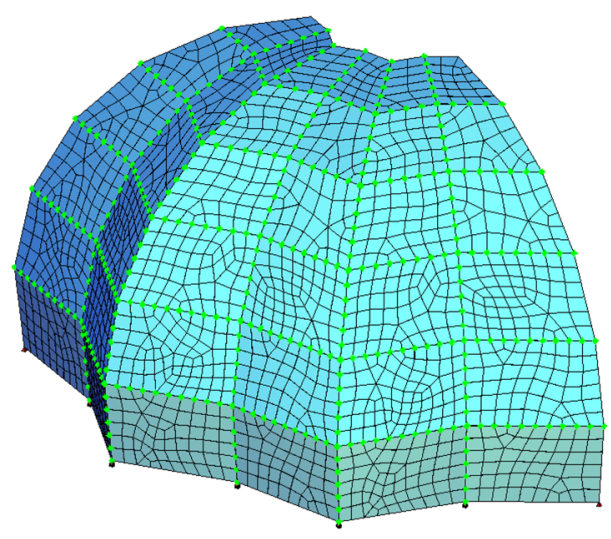

(a) The shell FEM model connected with spring hinges

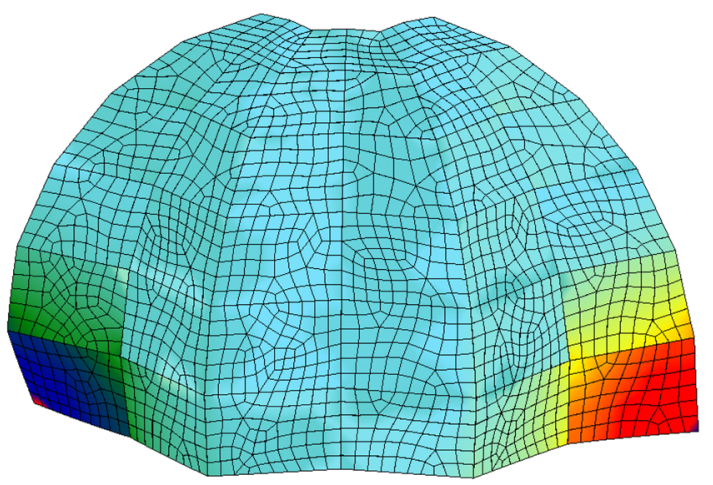

(b) The lowest eigen buckling mode

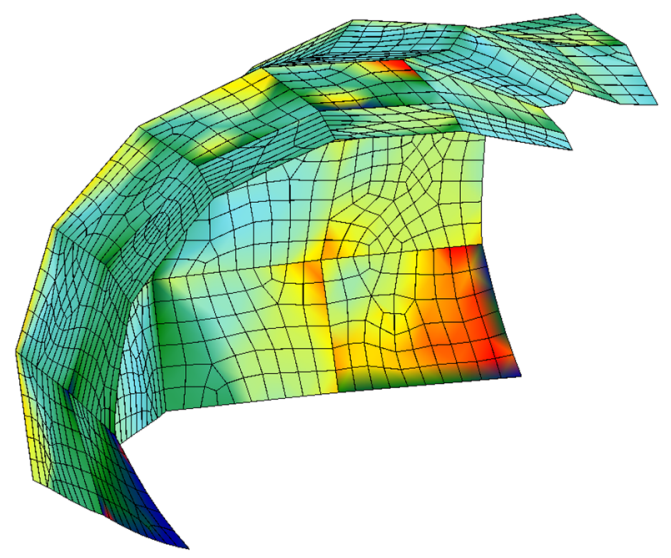

(c) Deformation under limit load by incremental load analysis (deformation amplified by a factor of 5)

Fig. 12 Stability analysis with thin shell finite element model with the initial configuration range for the angle to include the steeper fold and increasing the importance weighting of the objective function for structural stiffness. The resulting shape has a smaller angle $\alpha$ as seen in Fig. 13a). The linear joint stiffness for "watoji" lacing was introduced as defined in Table 1. The overall height was reduced from $3.0 \mathrm{~m}$ to $2.85 \mathrm{~m}$ by uniformly scaling the model, which was also deemed more appropriate in consideration of reducing the package size for transportation.

The lowest eigenvalue buckling factor was significantly increased from the initial model to 1.08, the buckling mode of which is shown in Fig. 13b). However, the incremental loading analysis indicated that geometric nonlinearity still caused instability with a limit load factor of 0.49 , which is about half of its self-weight. Figure 13c) shows the deformation under the limit loading. It was observed that the end bottom panels still buckle, but the propagation was localized only to the outer strips.

\subsubsection{Analysis results of the final geometry}

In order to mitigate instability and prevent such progressive deformation, web stiffener elements were introduced between valleys on the exterior side of the canopy at three levels as shown in Fig. 14a. Other configurations remain the same with the model described in the Sect. 5.2.2. The analysis shows that they stabilized the structure significantly, such that local buckling of the end bottom panels was prevented, and the lowest eigenvalue factor increased to 1.13. As each web consists of two triangle pieces linked with hinges combined with "watoji" lacing, they can fold in during closing transformation and function only in tension in the expanded position. They keep the folded plate system from flattening under its own weight as shown in Fig. 14c). A load limit factor of 1.32 by the output of incremental loading analysis with geometric nonlinearity is higher than the lowest factor of eigenvalue buckling analysis. This implies that the structure can undergo post-buckling behavior beyond the linear buckling load by reconfiguring the geometry to an updated equilibrium state. By correctly understanding the critical cause of the unstable behavior, the stabilizing elements were successfully accommodated in the folding system while increasing stability during deployment. Figure $14 d$ ) shows the actual implementation of the stabilizing webs.

\section{Materials and fabrication}

This section describes the fabrication of the canopy with a focus on the FRP hinges, the cutting pattern, module making, and the connection details for on-site assembly. 


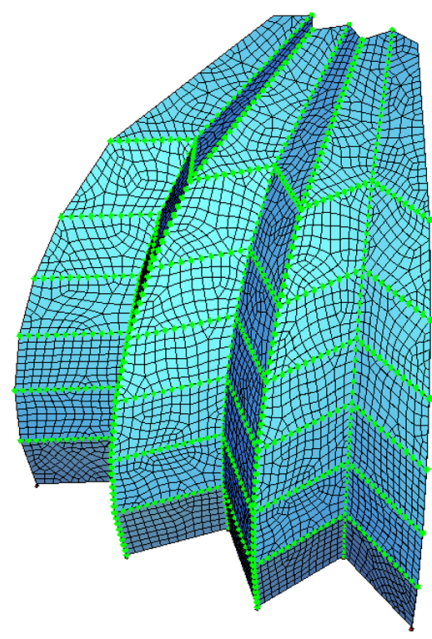

(a) The shell FEM model connected with spring hinges

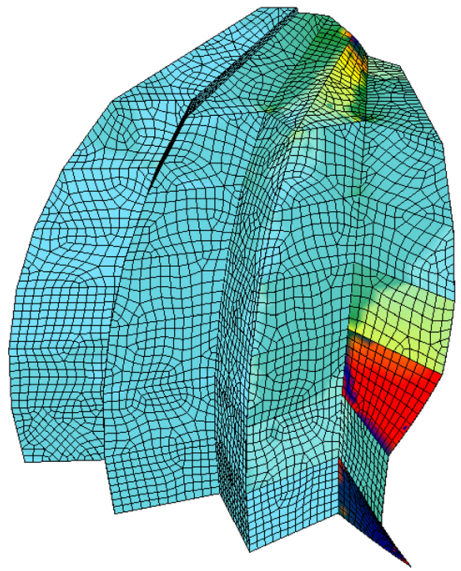

(b) The lowest eigen buckling mode

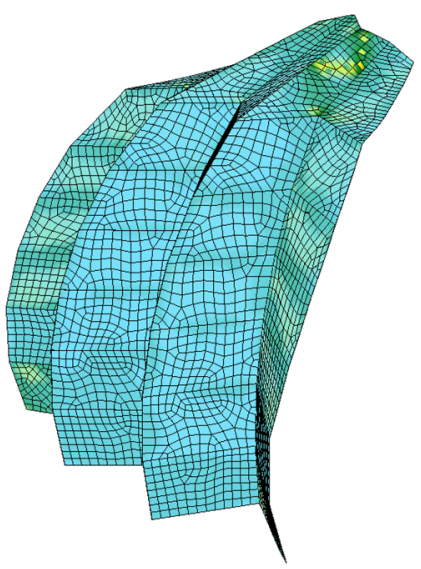

(c) Deformation under limit load by incremental load analysis (deformation amplified by a factor of 10 )

Fig. 13 Stability analysis with thin shell finite element model with the modified configuration

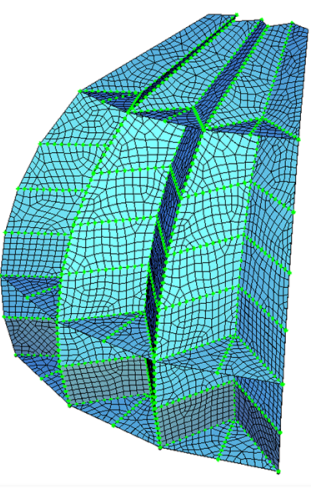

(a) The shell FEM model connected with spring hinges

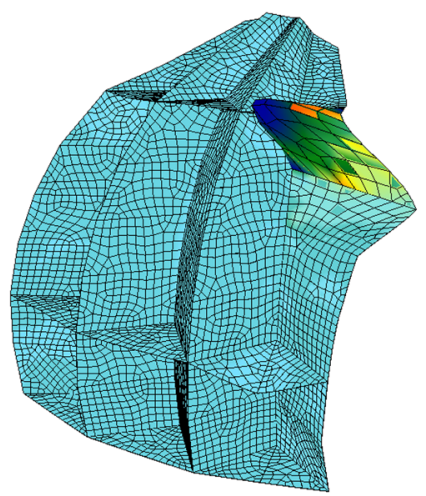

(b) The lowest eigen buckling mode

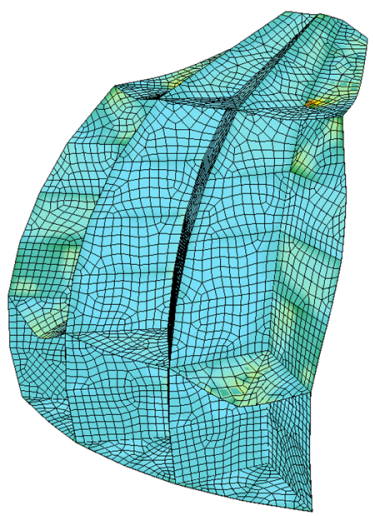

(c) Deformation under limit load by incremental load analysis

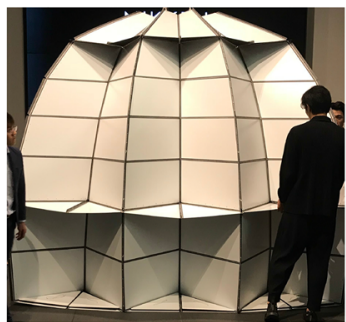

(d) Actual application of stiffening webs

Fig. 14 Stability analysis with thin shell finite element model with the stabilizing webs 
Figure 15 shows a perspective view and drawings of the fabricated canopy.

\subsection{FRP hinge}

The origami structure is formed using foldable Fiber Reinforced Plastics (FRP) using both carbon and grass fibers. The foldable FRP sheet consists of panel parts and joint parts. The panel parts are made from Carbon Fiber Reinforced Plastics (CFRP) and the joint parts consist of Grass Fiber Reinforced Plastics (GFRP) with embedded FRP hinges. Here, the FRP hinge is defined as a foldable line in which matrix resin is selectively replaced by a flexible rubber commonly used in composite structures. FRP hinges have already been investigated by Lopez et.al [5] and Todoroki et. al.[6]. The reinforced fiber is continuous throughout the composite structure including the foldable line, which ensures excellence in the mechanical reliability of the FRP hinge.

When the FRP hinge behaves as an elastic body, its contour shape at an arbitrary bending is described by "Elastica" theory and formulated by using the elliptic integral of the first order with the breadth of the hinge as a parameter (Fig. 16). For the actual FRP hinge, the bending force is lower for different orders of magnitude compared to the theoretical value. The attainable curvature also exceeds the expected level from the elongation of the break of the reinforced fiber, although the apparent contour profile is very similar to the theoretical one. These phenomena are explained by micro-buckling which is attributable to shear deformation of the rubber component under compression [20]. The micro-buckling had no significant effects on the folding endurance of the FRP hinge in this case.

For Origami structure using thick rigid panels, the spatial interference between adjacent panels could hamper desired folding motions. For FRP hinges, the problem can be overcome by suitable choice of the hinge breadths.

\subsection{Cutting pattern and module manufacture}

The canopy consists of deployable strips and webs. This section describes the cutting pattern and module manufacture process. Because each strip is approximately three meters long and two meters wide, they must be divided into smaller modules in consideration of cost and ease of fabrication and transportation. The modules should be less than $1.0 \times 3.0 \mathrm{~m}$ whilst there should also not be more than three cutting modules for easy assembly. Under these requirements, the cutting modules are determined as follows (Fig. 17).
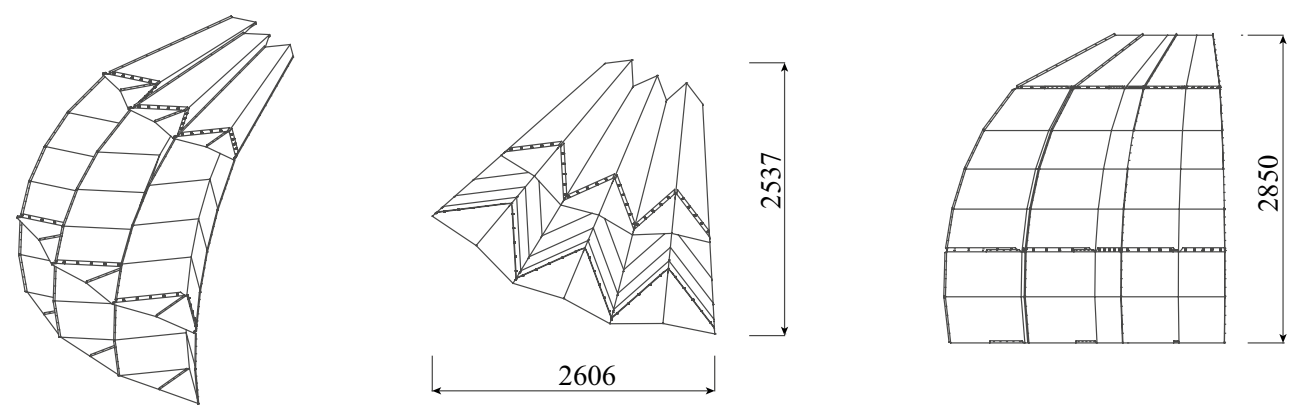

Fig. 15 Perspective, top, and front views of the fabricated canopy

Fig. 16 The calculated contour profile of the FRP hinge at bending angle a based on Elastica theory

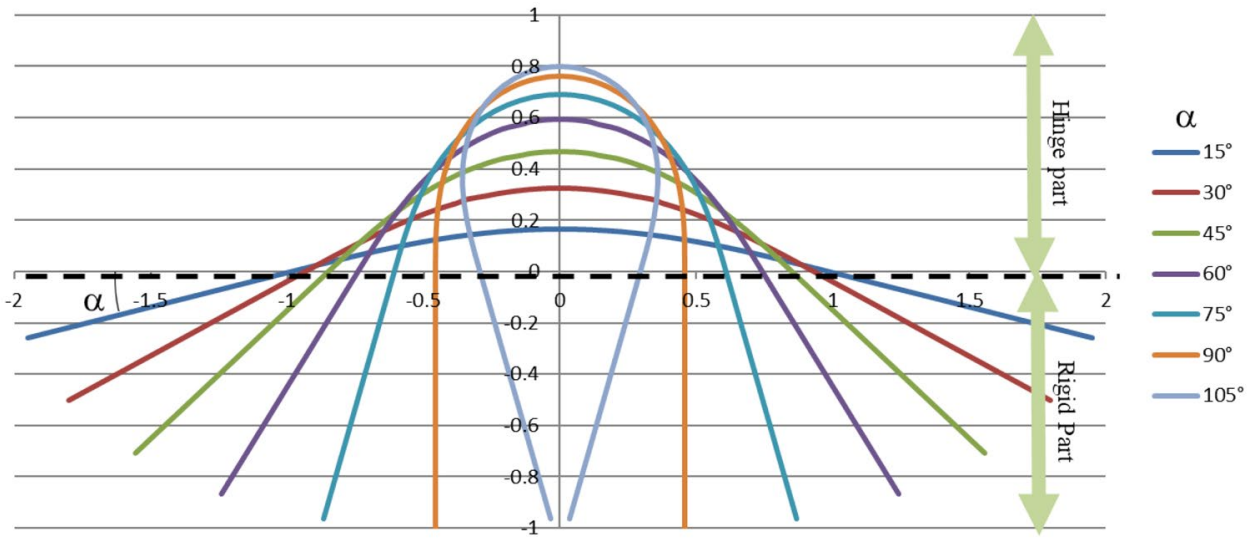



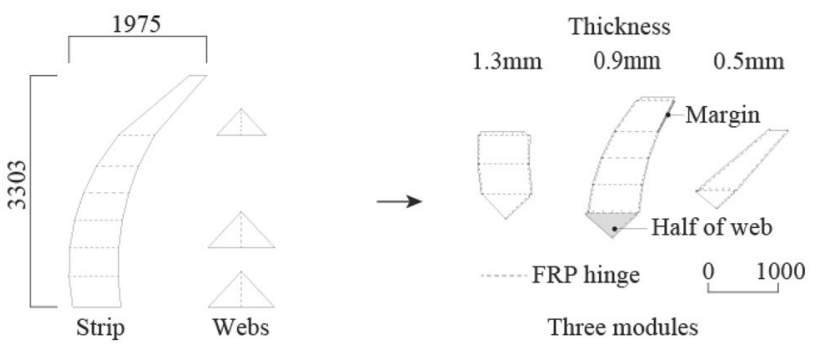

Fig. 17 Cutting pattern

The number of the modules is set to three because of the maximum size allowed. A half of each web is integrated to the lower side of each module. The margins for on-site assembly are integrated to the edges of the modules. The webs and margins are integrated to the module via above-mentioned FRP hinges.

The deployable modules have two types of FRP hinges. The ones along the horizontal foldable lines are integrated between panels in each module, with a required fold angle of about $15^{\circ}$. On the other hand, those along the vertical foldable lines are integrated with the assembling margins, which are in turn connected to adjacent margins to complete the linear joint for folding. The required fold angle is about $50^{\circ}$. A higher rotational stiffness is preferred in the horizontal hinges as they more significantly impact structural stability. Lowering rotational stiffness is preferred in the vertical hinge for deployment. Therefore, the hinge breadth of the horizontal and the vertical flange were set to $4 \mathrm{~mm}$ and $8 \mathrm{~mm}$, respectively.

Figure 18 shows the process flow for the prototyping based on the hand-lay-up method. The flange parts with the FRP hinge were separately prepared and finally combined with the main body. For the flange parts CF-epoxy prepregs were stacked on both sides of a dry glass fabric, which is used as a core (except for the foldable line areas), and then silicon resin (KE-4S-TS, Shin-etsu) suffused into them, followed by vacuum bagging and curing in an autoclave at $130^{\circ} \mathrm{C}$ under $0.15 \mathrm{MPa}$. For the main body, each panel was prepared by lamination of the CF prepregs, connected with the finished flange by using bonding film and cured in the same way as described above. The main body and the flange parts were completely consolidated by the curing process.

\subsection{Connection detail for on-site assembly}

"Watoji" joints, a traditional Japanese book binding technique [21] as shown in Fig. 19, are applied to the connection between modules. This joint has several remarkable properties beside it's tightness: (1) it is reusable as it does not use bonding material; (2) it can be assembled and disassembled by hand; (3) it is relatively tolerant to manufacturing errors; and, (4) it goes well with origami concepts as both were developed for paper art works. The string
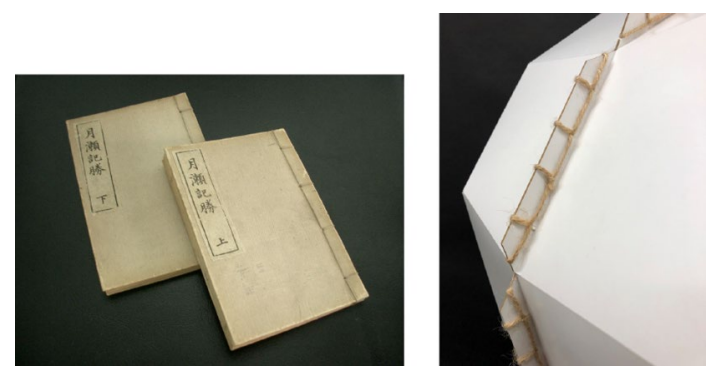

Fig. 19 Watoji joint in book binding (left) applied to $1 / 2$ scale model (right)
Fig. 18 The process flow chart for prototyping the foldable Origami structure
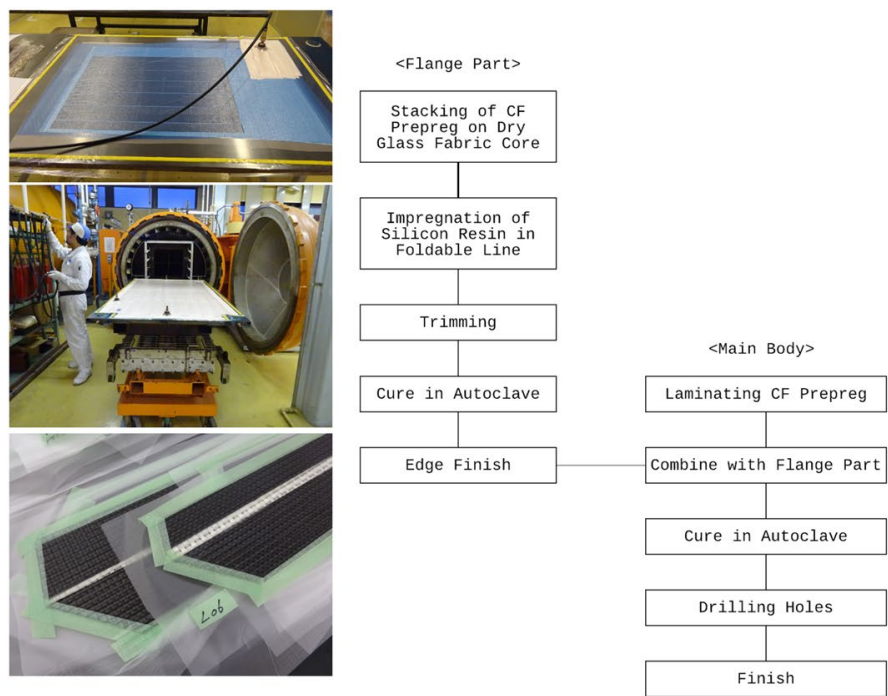
made of PET is utilized at the connections for its strength and durability.

\section{Conclusion and future vision}

This paper has proposed an origami-based lightweight deployable structure made of fiber-reinforced composite materials with embedded panels and compliant hinges. We have shown the design process including the development of the geometric concept based on rigid origami, the multi-objective optimization scheme for preliminary design of the initial geometry, sophisticated structural analyses to investigate the kinematic behaviors and instability issues, and the integration to fabrication process made by only planar pieces. Several different mathematical models were developed to address these subjects respectively. Analysis with different models were repeatedly performed to feed and integrate the findings from each investigation into the final design.

Through the stability analysis with the geometric nonlinear shell finite elements, the authors were able to identify the critical buckling behaviors of the rigid origami structure, which is made of extremely thin panels articulated with very flexible hinges. Furthermore, through dynamic structural analysis and singular value decomposition of the polyhedralized truss system, the authors identified kinematic bifurcation in the structure, which is not always evident through the study with an origami simulation model alone. This phenomenon should be further investigated as it concerns the control of transformable structures.

The folding structure was fabricated by the manual assembly of flange parts with hinges followed by a second curing. This particular process was chosen because of the limitation of available materials and equipment at the time. However, ideally, this hinge placement process can be replaced by 2-axis CNC or printing techniques, so that we may pattern the creases composed of soft and hard matrix by using a single fiber fabric. We envision that our origami-based approach can lead to developments in automated computational 3D forming based on folding techniques in the future.

Acknowledgements The authors would like to thank the support from Nikken Sekkei and Teijin. The last author is supported by Japan Society for the Promotion of Science KAKENHI grant number $16 \mathrm{H} 06106$. The authors would also like to thank Wasim llyas for the help in English editing

Funding This work is supported by Nikken Sekkei, Teijin, and Japan Society for the Promotion of Science KAKENHI grant number $16 \mathrm{H} 06106$.

\section{Compliance with ethical standards}

Conflict of Interest On behalf of all authors, the corresponding author states that there is no conflict of interest except listed in the funding section.

Open Access This article is licensed under a Creative Commons Attribution 4.0 International License, which permits use, sharing, adaptation, distribution and reproduction in any medium or format, as long as you give appropriate credit to the original author(s) and the source, provide a link to the Creative Commons licence, and indicate if changes were made. The images or other third party material in this article are included in the article's Creative Commons licence, unless indicated otherwise in a credit line to the material. If material is not included in the article's Creative Commons licence and your intended use is not permitted by statutory regulation or exceeds the permitted use, you will need to obtain permission directly from the copyright holder. To view a copy of this licence, visit http://creativecommons .org/licenses/by/4.0\%.

\section{References}

1. Hoberman CS (1988) Reversibly expandable three-dimensional structure. U.S. Patent No. 4,780,344. Washington, DC: U.S. Patent and Trademark Office

2. Tachi T (2009a) Generalization of rigid-foldable quadrilateralmesh origami. J Int Assoc Shell Spat Struct 50(3):173-179

3. Tachi T, Miura K (2012) Rigid-foldable cylinders and cells. J Int Assoc Shell Spat Struct 53(4):217-226

4. Tachi T (2019) Introduction to structural origami. J Int Assoc Shell Spat Struct 60(1):7-18

5. Lopez JF, Pellegrino S (2009) Folding of thin-walled composite structures with a soft matrix. In Proceedings of 50th AIAA/ASME/ ASCE/AHS/ASC structures, structural dynamics, and materials conference, Palm Springs, CA, 2009

6. Todoroki A, Kumagai K, Matsuzaki R (2009) Self-deployable space structure using partially flexible CFRP with SMA wires. J Intell Mater Syst Struct 20:1415-1424

7. Saito K, Pellegrino S, Nojima T (2014). Manufacture of arbitrary cross-section composite honeycomb cores based on origami techniques. J Mech Des 136(5):051011

8. Al-Mansoori M, Khan KA, Cantwell WJ (2020) Harnessing architected stiffeners to manufacture origami-inspired foldable composite structures. Compos Sci Technol 200:108449

9. Filipov ET, Paulino GH, Tachi T (2016) Origami tubes with reconfigurable polygonal cross-sections. Proc R Soc A 472:20150607

10. Vierlinger R (2017) Octopus. https://www.food4rhino.com/app/ octopus/.Accessed 2017

11. Bader J, Zitzler E (2008) HypE: an algorithm for fast hypervolume-based many-objective optimization. TIK report 286. Computer Engineering and Networks Laboratory, ETH Zurich

12. Karamba3d: https://www.karamba3d.com/

13. Argyris JH, Papadrakakis $M$, Apostolopoulou C, Koutsourelakis S, Koutsourelakis P-S (2000) The TRIC shell element: theoretical and numerical investigation. Comput Methods Appl Mech Eng 182(1-2):217-245

14. https://github.com/bunjii/reflection

15. Sadeghipour Roudsari, M., Mackey, C.: Ladybug tools. https:// www.ladybug.tools/ (2017)

16. Tachi $T(2009 \mathrm{~b})$ Simulation of rigid origami Origami 4(08):175-187 
17. Resch R, Christiansen HN (1970) Kinematic folded plate system. In IASS symposium on Folded Plates and Prismatic Structures

18. Filipov ET, Liu K, Tachi T, Schenk M, Paulino GH (2017) Bar and hinge models for scalable analysis of origami. Int J Solids Struct 124:26-45

19. Tachi T (2010) Freeform variations of origami. J Geom Graph 14(2):203-215

20. Jiménez FL, Pellegrino S (2012) Folding of fiber composites with a hyperelastic matrix. Int J Solids Struct 49(3-4):395-407

21. user: Excl-zoo / wikimedia commons / public domain https:// commons.wikimedia.org/wiki/File:Tsukigase-Kisho-Manuscript -Books.jpg

22. Crhisfield MA. Non-linear finite element analysis of solids and structures volume 1. Essentials, Wiley

23. Liu X, Gattas JM, Chen Y (2016) One-DOF superimposed rigid origami with multiple states. Sci rep 6:36883

24. Tachi T, Hull TC (2017). Self-foldability of rigid origami. J Mech Robot 9(2):021008

25. Filipov ET, Tachi T, Paulino GH (2015) Origami tubes assembled into stiff, yet reconfigurable structures and metamaterials. Proc Natl Acad Sci 112(40):12321-12326
26. Chen BGG, Liu B, Evans AA, Paulose J, Cohen I, Vitelli V, Santangelo CD (2016) Topological mechanics of origami and kirigami. Phys Rev Lett 116(13):135501

27. Silverberg JL, Na JH, Evans AA, Liu B, Hull TC, Santangelo CD, Cohen I (2015) Origami structures with a critical transition to bistability arising from hidden degrees of freedom. Nat Mater 14(4):389-393

28. Liu K, Paulino GH (2017) Nonlinear mechanics of non-rigid origami: an efficient computational approach. Proc R Soc A Math Phys Eng Sci 473(2206):20170348

29. SOFiSTiK Manual: ASE general static analysis of finite element structures (2018)

Publisher's Note Springer Nature remains neutral with regard to jurisdictional claims in published maps and institutional affiliations. 